\title{
TEORIA FUNDAMENTADA NOS DADOS EM TESES E DISSERTAÇÕES DA ENFERMAGEM BRASILEIRA ${ }^{1}$
}

\author{
Glauber Weder dos Santos Silva², Bertha Cruz Enders³, Francisca Georgina Macedo de Sousa ${ }^{4}$, Julliana \\ Fernandes de Sena ${ }^{5}$, Renata Clemente dos Santos ${ }^{6}$, Amanda Barbosa da Silva $^{7}$
}

${ }^{1}$ O presente trabalho foi realizado com apoio da Coordenação de Aperfeiçoamento de Pessoal de Nível Superior - Brasil (CAPES) - Código de Financiamento 001

${ }^{2}$ Doutorando do Programa de Pós-Graduação em Enfermagem (PGEnf) da Universidade Federal do Rio Grande do Norte (UFRN). Professor Temporário da Faculdade de Ciências da Saúde do Trairí da UFRN. Natal, Rio Grande do Norte, Brasil. E-mail: glauberweder@hotmail.com

${ }^{3}$ Doutora em Enfermagem. Professora do PGEnf/UFRN. Natal, Rio Grande do Norte, Brasil. E-mail: berthacruz.enders@gmail.com

${ }^{4}$ Doutora em Enfermagem. Professora do PGEnf da Universidade Federal do Maranhão. São Luís, Maranhão, Brasil. E-mail: fgeorginasousa@hotmail.com

${ }^{5}$ Mestranda do PGEnf/UFRN. Natal, Rio Grande do Norte, Brasil. E-mail: jullianafsena@yahoo.com.br

${ }^{6}$ Mestranda do PGEnf/UFRN. Natal, Rio Grande do Norte, Brasil. E-mail: renata.clemente@hotmail.com

${ }^{7}$ Mestranda do PGEnf/UFRN. Natal, Rio Grande do Norte, Brasil. E-mail: amandab641@hotmail.com

\section{RESUMO}

Objetivo: identificar como os pesquisadores utilizaram o método da Teoria Fundamentada nos Dados nas teses e dissertações da enfermagem brasileira.

Método: trata-se de estudo documental com abordagem quantitativa. Como fonte de coleta de dados, foram utilizados os relatórios depositados no Catálogo de Teses e Dissertações do Centro de Estudos e Pesquisas em Enfermagem da Associação Brasileira de Enfermagem, entre 2001 e 2015, com posterior resgate da versão completa no repositório da instituição de ensino superior de origem das teses e dissertações. A coleta ocorreu a partir de extração dos dados em planilhas que foram submetidos à analise descritiva.

Resultados: elegeram-se 70 teses e dissertações (0,95\%) do total de 7.369 estudos. A maioria (70\%) descreveu como foram construídos os grupos amostrais. Somente $4,3 \%$ não registrou ter realizado a coleta e analise dos dados de forma concomitante. Dos estudos que descreveram todas as etapas, $34,3 \%$ não apresentaram o processo de validação do modelo teórico. Os diagramas e os memorandos foram utilizados, respectivamente, em 74,3\% e 65,7\% das teses e dissertações analisadas. O referencial teórico mais frequente foi o Interacionismo Simbólico e a perspectiva metodológica foi a Straussiana. Evidenciou-se que para os pesquisadores, a entrevista foi a principal técnica de coleta utiliza de forma isolada e combinada com outras abordagens.

Conclusão: a diversidade na apresentação e na descrição do método e resultados dos estudos revela que ainda há necessidade de aprimoramento quanto ao uso do método, considerando as diferentes vertentes da teoria fundamentada nos dados.

DESCRITORES: Pesquisa em enfermagem. Pesquisa metodológica em enfermagem. Pesquisa qualitativa. Teoria fundamentada. Educação de pós-graduação em enfermagem.

\section{GROUNDED THEORY IN THESES AND DISSERTATIONS OF BRAZILIAN NURSING}

\footnotetext{
ABSTRACT

Objective: to identify how the researchers used the method of Grounded Theory in theses and dissertations of the Brazilian nursing. Method: this is a documentary study with a quantitative approach. As a source of data collection, the reports deposited in the Catalog of Theses and Dissertations of the Center for Nursing Studies and Research of the Brazilian Nursing Association between 2001 and 2015 were used, with subsequent redemption of the full version in the repository of the higher education institution of theses and dissertations. The data collection was carried out from data extraction in spreadsheets that were submitted to the descriptive analysis.

Results: 70 theses and dissertations $(0.95 \%)$ were selected from the total of 7,369 studies. The majority $(70 \%)$ described how the sample groups were constructed. Only $4.3 \%$ did not report having collected and analyzed the data concomitantly. From the studies that described all the steps, $34.3 \%$ did not present the validation process of the theoretical model. The diagrams and memos were used, respectively, in $74.3 \%$ and $65.7 \%$ of the theses and dissertations analyzed. The most frequent theoretical reference was Symbolic Interactionism and the methodological perspective was the Straussian. It was evidenced that for the researchers, the interview was the main collection technique used in isolation and combined with other approaches.

Conclusion: the diversity in the presentation and description of the method and results of the studies reveals that there is still need for improvement regarding the use of the method, considering the different aspects of the Grounded Theory.
} 


\title{
TEORÍA FUNDAMENTADA EN LOS DATOS EN TESIS Y DISERTACIONES DE LA ENFERMERÍA BRASILEÑA
}

\begin{abstract}
RESUMEN
Objetivo: identificar cómo los investigadores utilizaron el método de la Teoría Fundamentada en los Datos en las tesis y disertaciones de la enfermería brasileña.

Método: se trata de un estudio documental con enfoque cuantitativo. Como fuente de recolección de datos, se utilizaron los informes depositados en el Catálogo de Tesis y Disertaciones del Centro de Estudios e Investigaciones en Enfermería de la Asociación Brasileña de Enfermería, entre 2001 y 2015, con posterior rescate de la versión completa en el repositorio de la institución de enseñanza superior de origen de las tesis y disertaciones. La recolección ocurrió a partir de extracción de los datos en hojas que fueron sometidos al análisis descriptivo.

Resultados: se eligieron 70 tesis y disertaciones (0,95\%) del total de 7.369 estudios. La mayoría (70\%) describió cómo se construyeron los grupos de muestra. Sólo el 4,3\% no registró haber realizado la recolección y análisis de los datos de forma concomitante. De los estudios que describieron todas las etapas, el 34,3\% no presentó el proceso de validación del modelo teórico. Los diagramas y los memorandos se utilizaron respectivamente en el 74,3\% y el 65,7\% de las tesis y disertaciones analizadas. El referencial teórico más frecuente fue el Interaccionismo Simbólico y la perspectiva metodológica fue la Straussiana. Se evidenció que para los investigadores, la entrevista fue la principal técnica de recolección utiliza de forma aislada y combinada con otros enfoques.

Conclusión: la diversidad en la presentación y en la descripción del método y resultados de los estudios revela que todavía hay necesidades de perfeccionamiento en cuanto al uso del método, considerando las diferentes vertientes de la teoria fundamentada em los dados.

DESCRIPTORES: Investigación en enfermería. Investigación metodológica en enfermería. Investigación cualitativa. Teoría fundamentada. Educación de postgrado en enfermería.
\end{abstract}

\section{INTRODUÇÃO}

A enfermagem brasileira vem aliando diferentes domínios e referenciais metodológicos a fim de garantir rigor científico em sua produção, tornando possível elucidações do objeto investigado a partir do melhor enquadramento teórico-filosófico para apreender as raízes de problemas estudados. ${ }^{1}$ Assim, tomam evidência as produções oriundas de Programas de Pós-Graduação (PPG) em Enfermagem, como importante cenário do saber científico e tecnológico que resultam em estudos que contribuem diretamente para a qualidade da assistência, do cuidado e da credibilidade profissional. ${ }^{2}$

Neste contexto, nas últimas décadas, houve um aumento considerado de pesquisas com abordagem qualitativa, principalmente na comunidade científica das ciências da saúde, empoderando-se de um conhecimento proveniente do campo das ciências sociais, abordando aspectos do mundo social e complexo. ${ }^{3}$ No Brasil, a enfermagem, enquanto disciplina acadêmica das ciências da saúde, foi pioneira na incorporação de métodos interacionistas e fenomenológicos, os quais se tornaram, com o passar dos anos, importantes ferramentas metodológicas para se construir o corpus de conhecimento da profissão. ${ }^{4}$

Na pesquisa qualitativa, investe-se no aprofundamento sobre entidades e processos do contexto cultural e social e seus significados, que não podem, naturalmente, ser quantificados. É uma íntima relação entre pesquisador e objeto que realçam e aproximam a experiência e as relações sociais, envolvendo-se numa natureza naturalista, interpretativa, criativa e flexível na verificação de fenômenos complexos, objetivando sempre descrevê-los ou compreendê-los. ${ }^{5}$

Oportunamente, a Grounded Theory, chamada no Brasil de Teoria Fundamentada nos Dados (TFD), entre outras abordagens metodológicas de caráter qualitativo, surge como metodologia emergente na pesquisa em enfermagem. Tratase de um método indutivo e dedutivo de análise comparativa a partir de um conjunto de procedimentos que geram, a partir do aprofundamento, uma teoria embasada nos dados. ${ }^{6}$ Assim, o principal objetivo da TFD é compreender as experiências do mundo empírico e as interações entre os sujeitos que o compõem em um determinado contexto social, evidenciando determinado processo social ou psicossocial, obtendo, para isso, uma teoria que apresente estratégias desenvolvidas diante situações vivenciadas. ${ }^{6-7}$

Na TFD incorpora-se, portanto, a interação e a introspecção do comportamento humano, pois prioriza uma dimensão subjetiva capaz de orientar ações do cuidado de enfermagem, boas práticas de saúde e implementar processos multifocais e complexos. ${ }^{8}$ Não obstante, requer, para seu desenvolvimento, versatilidade e rigor na apresentação 
dos fatos analisados, obedecendo criteriosamente os passos metodológicos propostos pelos autores.

No entanto, apresenta-se como desafio o emprego do rigor para se criar uma Teoria embasada nos dados, sobretudo em ambientes acadêmicos positivistas. Assim, justifica-se este estudo documental quanto à heterogeneidade com que os relatórios de pesquisas com TFD têm sido apresentados nas produções brasileiras, principalmente quanto aos diferentes recursos analíticos utilizados pelos autores em virtude das diferentes posições de publicações acadêmicas, por exemplo: recursos de coleta de dados, etapas de codificação e validação. ${ }^{7-8}$

Com base no exposto, o presente estudo elegeu as seguintes questões norteadoras: como o método TFD foi operacionalizado nas dissertações e teses disponíveis no Catálogo de Teses e Dissertações da Associação Brasileira de Enfermagem? Quais recursos analíticos vêm sendo utilizados nos estudos científicos de enfermagem com TFD?

Neste sentido, a fim de contribuir para elucidação das questões relacionadas com o desenvolvimento de estudos com TFD, o objetivo deste estudo foi identificar como os pesquisadores utilizaram o método da Teoria Fundamentada nos Dados nas teses e dissertações da enfermagem brasileira.

\section{MÉTODO}

Trata-se de estudo de abordagem quantitativa, do tipo documental, descritivo e comparativo, a partir do mapeamento das produções nos cursos stricto senso dos Programas de Pós-Graduação em Enfermagem no Brasil que utilizaram o método da TFD. O estudo documental consiste na análise de material que não recebeu tratamento científico, como por exemplo, os relatórios, objetivando extrair informações para análise técnica. ${ }^{9}$

Dessa forma, utilizou-se como fonte para coleta de dados o Catálogo de Teses e Dissertações do Centro de Estudos e Pesquisas em Enfermagem da Associação Brasileira de Enfermagem (CEPEn/ ABEn), que documenta títulos e resumos da produção nos Programa de Pós-Graduação em Enfermagem no Brasil. A escolha por tal material se deu por considerá-lo o mais consistente entre a produção de enfermagem na pesquisa científica, sendo essa modalidade de trabalhos caracterizada pela variância temática, criatividade, rigorosidade e logicidade, elementos pertinentes e essenciais para o desenvolvimento metodológico de uma pesquisa com TFD. Ademais, optou-se por realizar a pesquisa utilizando teses e dissertações, considerando que nesse tipo de relatório de pesquisa a descrição metodológica é mais completa do que o encontrado em artigos publicados.

A coleta de dados ocorreu entre junho ejulho de 2016, realizada por três mestrandas e um doutorando. Para facilitar a organização e registro dos dados pelos pesquisadores, elaborou-se o Protocolo de Pesquisa Documental, composto pelos seguintes itens: tema, objetivo do estudo, questão norteadora e estratégias de busca quanto à base de dados, critérios de seleção dos estudos e coleta e síntese de dados.

Utilizou-se como fonte de coleta de dados os volumes de XIX a XXXIII do CEPEn/ABEn, compreendendo as publicações do recorte temporal entre os anos 2001 a 2015, obedecendo aos seguintes critérios de inclusão: (1) ser dissertações ou teses; (2) estar vinculado aos Programa de Pós-Graduação em Enfermagem do Brasil; e, (3) ter a TFD como recurso metodológico.

Na primeira fase do estudo, para se identificar as dissertações e teses que utilizaram a TFD, selecionou-se a produção por meio de leitura dos títulos e resumos contidos no CEPEn/ABEn e, na segunda, por leitura integral dos relatórios de pesquisa. Ao se identificar, a partir dos títulos e resumos, a produção de dissertações e teses da enfermagem com TFD, pesquisaram-se os relatórios nos repositórios institucionais onde estas foram defendidas. Assim, excluíram-se as teses e dissertações que não estavam disponíveis eletronicamente na íntegra nos bancos de dados e repositórios das Instituições de Ensino Superior (IES) de origem.

Os dados coletados e organizados em planilha do Microsoft Office Excel ${ }^{\circledR}$ foram submetidos à análise das frequências absolutas e relativas, segundo as variáveis: Formação do autor (formação acadêmica em nível de graduação do autor, consultada via Plataforma Lattes); Nível acadêmico (doutorado, mestrado acadêmico e mestrado profissional); Instituição de ensino (IES na qual vincula-se a pesquisa); Ano de defesa da tese ou dissertação; Local de desenvolvimento do estudo (unidade federativa onde se realizou a coleta de dados da pesquisa); Perspectiva metodológica da TFD (classificação dos trabalhos nas três principais vertentes metodológicas que surgiram ao longo do percurso evolutivo do método - Clássica, Straussiana e Construtivista), ${ }_{i}^{8}$ Referencial teórico (análise do referencial teórico que permite identificar a autonomia do conhecimento produzido e a relação com outras disciplinas do conhecimento científico); ${ }^{1}$ Área de conhecimento da enfermagem (agrupamento dos estudos nas categorias processuais do trabalho em enfermagem - assistência, ensino, pesquisa e gerência); e, Recursos analíticos 
(de acordo com os aspectos conceituais, estruturais e operativos da TFD, ${ }^{8}$ agrupando-se os resultados nas seguintes categorias - descrição da construção dos grupos amostrais, simultaneidade da coleta e análise dos dados, processo de codificação dos dados, uso de diagramas, uso de memorandos e, apresentação da teoria: modelo teórico/matriz teórica/teoria substantiva).

A fim de favorecer a análise, os dados das teses e dissertações serão apresentados em conjunto. Destaca-se que, por tratar-se de estudo documental pautado em dados públicos, a aprovação do Comitê de Ética e Pesquisa não se fez necessária.

\section{RESULTADOS}

No Catálogo de teses e dissertações do CEPEn/ABEn foram localizados 7.369 resumos, considerando os volumes XIX ao XXXIII. Destes, 70 estudos que utilizaram a TFD foram resgatados nos repositórios das IESs e selecionados para integrar a amostra final da pesquisa documental, que corresponde a $0,95 \%$ das pesquisas de enfermagem produzidas entre 2001 e 2015. A tabela 1 apresenta os dados quantitativos referentes às etapas de buscas dos estudos.

Expressivamente, os últimos cinco anos de publicações (2010-2014) somam 54,3\% (n=38) de toda produção analisada, em detrimento dos primeiros nove anos (2001-2009), que apesar de concentrarem valor aproximado (32; 45,7\%), é produto de maior recorte temporal.

Tabela 1 - Total de estudos nos Catálogos de Pesquisas e Pesquisadores de Enfermagem do CEPEn, com Teoria Fundamentada nos Dados, pré-selecionados e selecionados. Natal, RN, Brasil, 2016. (n=70)

\begin{tabular}{|c|c|c|c|c|c|c|c|c|}
\hline \multirow[t]{2}{*}{ Volumes } & \multicolumn{2}{|c|}{ Total de estudos* } & \multicolumn{2}{|c|}{$\begin{array}{c}\text { Estudos pré-seleciona- } \\
\text { dos } \dagger\end{array}$} & \multicolumn{2}{|c|}{ Estudos selecionados $\ddagger$} & \multicolumn{2}{|c|}{ Amostra final } \\
\hline & $\mathbf{n}$ & $\%$ & $\mathbf{n}$ & $\%$ & $\mathrm{n}$ & $\%$ & $\mathbf{n}$ & $\%$ \\
\hline XIX & 469 & 6,36 & 5 & 4,55 & 2 & 2,30 & 2 & 2,86 \\
\hline$x X$ & 280 & 3,80 & 4 & 3,64 & 3 & 3,45 & 3 & 4,29 \\
\hline XXI & 285 & 3,87 & 2 & 1,82 & - & - & - & - \\
\hline XXII & 318 & 4,32 & 7 & 6,36 & 1 & 1,15 & 1 & 1,43 \\
\hline XXIII & 383 & 5,20 & 5 & 4,55 & 1 & 1,15 & 1 & 1,43 \\
\hline XXIV & 554 & 7,52 & 9 & 8,18 & 8 & 9,20 & 8 & 11,43 \\
\hline XXV & 518 & 7,03 & 6 & 5,45 & 5 & 5,75 & 4 & 5,71 \\
\hline XXVI & 445 & 6,04 & 5 & 4,55 & 5 & 5,75 & 5 & 7,14 \\
\hline XXVII & 482 & 6,54 & 8 & 7,27 & 5 & 5,75 & 4 & 5,71 \\
\hline XXVIII & 368 & 4,99 & 7 & 6,36 & 4 & 4,60 & 4 & 5,71 \\
\hline XXIX & 547 & 7,42 & 10 & 9,09 & 9 & 10,34 & 7 & 10,00 \\
\hline$x x x$ & 900 & 12,21 & 18 & 16,36 & 17 & 19,54 & 14 & 20,00 \\
\hline XXXI & 806 & 10,94 & 8 & 7,27 & 11 & 12,64 & 6 & 8,57 \\
\hline XXXII & 565 & 7,67 & 4 & 3,64 & 6 & 6,90 & 4 & 5,71 \\
\hline XXXIII & 449 & 6,09 & 12 & 10,91 & 10 & 11,49 & 7 & 10,00 \\
\hline Total & 7369 & 100 & 110 & 100 & 87 & 100 & 70 & 100 \\
\hline
\end{tabular}

*Número de resumos componentes do volume; †Conforme critérios de inclusão; łConforme critérios de inclusão e exclusão.

Houve predominância nas pesquisas dos cursos de doutorado $(36 ; 51,4 \%)$, seguida pelas dissertações de mestrado acadêmico $(33 ; 47,1 \%)$ e número reduzido em mestrado profissional $(1 ; 1,4 \%)$.

A Figura 1 apresenta as IES onde as pesquisas foram desenvolvidas. Apenas doze Instituições realizaram estudos com TFD no período avaliado e no recorte amostral, destacando-se a Universidade Federal de Santa Catarina (22; 31,4\%), a Universidade de São Paulo (22; 31,4\%), a Universidade Federal do Rio de Janeiro $(8 ; 11,4 \%)$ e a Universidade Federal do Paraná (7; 10\%). Quando agrupadas por regiões federativas, predominaram as IESs do eixo Sul (30;
$42,9 \%)$ e Sudeste $(36 ; 51,4 \%)$, seguidas pelo Nordeste (4; $5,7 \%)$. Nenhuma produção foi encontrada em IESs das regiões Norte e Centro-Oeste. 


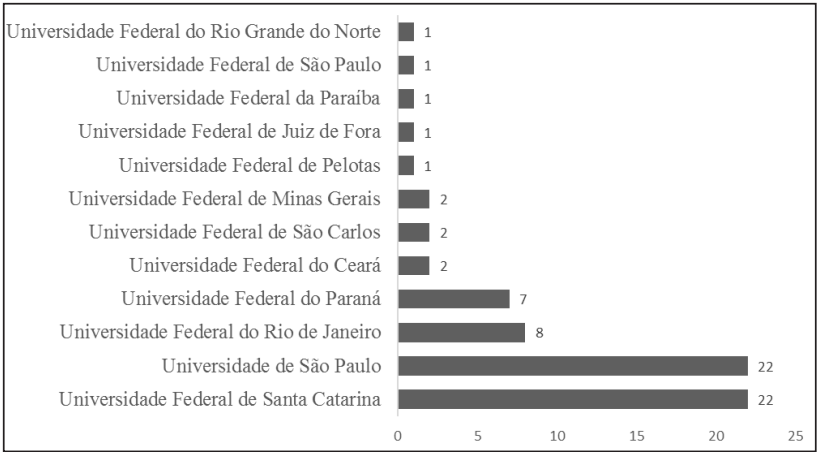

Figura 1 - Instituições de Ensino Superior segundo o número de teses e dissertações na área de Enfermagem, que utilizaram Teoria Fundamentada nos Dados. Natal, RN, Brasil, 2016. $(n=70)$
A maioria das pesquisas foi desenvolvida, ou seja, teve como lócus de coleta de dados, os Estados de São Paulo $(21 ; 30 \%)$, Santa Catarina $(18 ; 25,7 \%)$, Paraná $(9 ; 12,9 \%)$ e Rio de Janeiro $(8 ; 11,4 \%)$, concentrando-se nas IESs do eixo Sul e Sudeste.

Quanto à formação acadêmica em termos de graduação dos autores das pesquisas analisadas, estes são exclusivamente enfermeiros (70, 100\%), mesmo que algumas IES assumam caráter interdisciplinar em seus Programas de Pós-Graduação em Enfermagem.

A tabela 2 apresenta as áreas de conhecimento, referencial teórico e perspectivas metodológicas adotadas pelos estudos analisados.

Tabela 2 - Área de conhecimento de enfermagem, referencial teórico e perspectiva metodológica nas teses e dissertações com Teoria Fundamentada nos Dados. Natal, RN, Brasil, 2016. (n=70)

\begin{tabular}{|c|c|c|c|c|c|c|c|c|}
\hline \multicolumn{3}{|c|}{$\begin{array}{c}\text { Área de conhecimento de } \\
\text { Enfermagem }\end{array}$} & \multicolumn{3}{|c|}{$\begin{array}{c}\text { Referencial } \\
\text { teórico }\end{array}$} & \multicolumn{3}{|c|}{ Perspectiva metodológica } \\
\hline & $\mathbf{n}$ & $\%$ & & $\mathbf{n}$ & $\%$ & & $\mathbf{n}$ & $\%$ \\
\hline Assistência & 50 & 71,43 & Interacionismo simbólico & 48 & 68,57 & \multirow{4}{*}{$\begin{array}{l}\text { Straussiana } \\
\text { Clássica }\end{array}$} & \multirow{2}{*}{54} & \multirow{2}{*}{77,14} \\
\hline Pesquisa & 8 & 11,43 & \multirow{2}{*}{ Paradigma da complexidade } & \multirow{2}{*}{16} & \multirow{2}{*}{22,86} & & & \\
\hline Gerência & 8 & 11,43 & & & & & \multirow{2}{*}{16} & \multirow{2}{*}{22,86} \\
\hline Ensino & 4 & 5,71 & Não especificou & 6 & 8,57 & & & \\
\hline Total & 70 & 100 & Total & 70 & 100 & Total & 70 & 100 \\
\hline
\end{tabular}

Com relação às áreas de conhecimento da enfermagem, significativamente, predominaram as pesquisas sobre a prática assistencial $(71,4 \%)$. As dissertações e teses que abordaram a pesquisa e a gerência tiveram oito estudos cada (11,4\% para ambas). As de ensino representaram $5,7 \%$ da amostra $(n=4)$.

Ao analisar de forma conjunta o referencial teórico e a perspectiva metodológica que deram suporte à discussão e ao desenvolvimento da pesquisa, respectivamente, o Interacionismo Simbólico (48; $68,6 \%)$ e a vertente Straussiana $(54 ; 77,1 \%)$ destacaram-se, podendo essa preferência estar relacionada com a origem do método TFD. Paralelamente, o Paradigma da Complexidade $(16 ; 22,8 \%)$ e a vertente clássica $(16 ; 22,9 \%)$ surgem em seguida como suporte teórico e metodológico, respectivamente.

Quanto às técnicas de coleta de dados, 42 pesquisas $(58,3 \%)$ utilizaram mais de um recurso, conforme tabela 3. Houve predomínio no uso de entrevistas (100\%), sendo a do tipo semiestrutura a mais empregada $(91,4 \%)$. O recurso de observação $(88,1 \%)$ foi o mais empregado em combinação com a entrevista, predominando a observação não-participante $(52,4 \%)$.
Quanto aos aspectos conceituais, estruturais e operativos dos recursos analíticos descritos nas teses e dissertações, conforme tabela $4,70 \%$ apresentaram como foram construídos os grupos amostrais, importante estratégia para atender o princípio da amostragem teórica. Não descrever essa etapa, o que aconteceu em 30\% dos relatórios analisados, pode limitar a compreensão sobre o processo e decisões metodológicas adotadas pelo pesquisar.

Tabela 3 - Caracterização dos recursos utilizados para coleta de dados nas teses e dissertações com suporte da Teoria Fundamentada nos Dados. Natal, RN, Brasil, 2016. $(n=70)$

\begin{tabular}{|c|c|c|c|}
\hline Recurso & Tipo & $\mathbf{n}$ & $\%$ \\
\hline \multicolumn{4}{|c|}{ Utilizou apenas um recurso $(n=28 ; 40 \%)$} \\
\hline \multicolumn{4}{|c|}{ Entrevista } \\
\hline & Semiestruturada & 25 & 89,3 \\
\hline & Não-estruturada & 3 & 10,7 \\
\hline & Estruturada & 0 & 0 \\
\hline Total & & 28 & 100 \\
\hline \multicolumn{4}{|c|}{ Utilizou mais de um recurso $(n=42 ; 58,33 \%)$} \\
\hline \multicolumn{4}{|c|}{ Entrevista } \\
\hline & Semiestruturada & 39 & 92,9 \\
\hline & Não-estruturada & 2 & 4,8 \\
\hline
\end{tabular}




\begin{tabular}{llrr}
\hline \multicolumn{1}{c}{ Recurso } & \multicolumn{1}{c}{ Tipo } & n & \multicolumn{1}{c}{$\%$} \\
\hline \multirow{2}{*}{$\begin{array}{l}\text { Total } \\
\text { Observação }\end{array}$} & Estruturada & 1 & 2,3 \\
\multicolumn{1}{l}{ Total } & 42 & 58,33 \\
Documental & Não-participante & 22 & \\
Grupo focal & & 15 & 32,4 \\
\hline
\end{tabular}

Não atender à recomendação de coleta e análise simultânea dos dados $(4,3 \%)$ compromete o desenvolvimento dos conceitos a partir de suas dimensões e propriedades, isto é, categorias pouco desenvolvidas conceitualmente, embora 91,4\% dos pesquisadores tenham descrito todas as etapas do processo de codificação. Entretanto, 8,6\% omitiram uma destas etapas, relacionada à análise axial, isto é, a de consolidação de categorias e desenvolvimento do modelo paradigmático.

Tabela 4 - Caracterização das teses e dissertações quanto ao cumprimento dos processos analíticos da Teoria Fundamentada nos Dados. Natal, RN, Brasil, 2016. $(n=70)$

\begin{tabular}{|c|c|c|c|c|}
\hline \multirow{2}{*}{ Processos analíticos } & \multicolumn{2}{|c|}{ Sim } & \multicolumn{2}{|c|}{ Não } \\
\hline & $\mathbf{n}$ & $\%$ & $\mathbf{n}$ & $\%$ \\
\hline Descreveu o processo de construção de grupos amostrais & 49 & 70 & 21 & 30 \\
\hline A coleta e análise dos dados ocorreram simultaneamente & 67 & 95,7 & 3 & 4,3 \\
\hline Utilizou todas as etapas do processo de codificação & 64 & 91,4 & 6 & 8,6 \\
\hline Utilizou diagramas & 52 & 74,3 & 18 & 25,7 \\
\hline Utilizou memorandos & 46 & 65,7 & 24 & 34,3 \\
\hline Descreveu o processo de validação da teoria & 46 & 65,7 & 24 & 34,3 \\
\hline
\end{tabular}

Das que descreveram todas as etapas $(91,4 \%)$, expressivamente, 24 estudos $(34,3 \%)$ da amostra não validaram o construto teórico. Os diagramas e os memorandos foram utilizados $(74,3 \%$ e $65,7 \%$, respectivamente) nas teses e dissertações analisadas.

\section{DISCUSSÃO}

Mesmo diante dos avanços na disseminação da informação e do método em nível nacional, a sua utilização apresentou-se com pequena expressividade, corroborando com achados de uma revisão sobre o tema. ${ }^{10}$ Tal realidade pode ter relação com a progressiva expansão do uso método no território nacional e pouco domínio de alguns pesquisadores para sua execução diante das diferentes vertentes metodológicas. ${ }^{8}$

É importante também destacar que a TFD é divulgada nos corredores acadêmicos como método de difícil operacionalização. Sobre esse aspecto, é necessário desmitificar, pois o método apresenta estrutura metodológica que guia o pesquisador em todas as etapas, do processo de coleta à análise. Entretanto, ressalta-se que o pesquisador que faz opção pela TFD irá manejar grande volume de dados e a necessidade de utilizar concomitantemente a indução, a dedução, a sensibilidade e a criatividade de forma dinâmica e sistemática. Talvez, o aspecto exposto seja o que mais implique em dificuldades com o método.
Mediante os resultados da busca, elucidouse que, mais da metade das investigações que utilizaram TFD como método de pesquisa estão concentradas a partir do ano de 2010. Tal evidência pode estar associada à maior visibilidade em nível nacional a partir do ano de 2008, uma vez que as principais obras ${ }^{6-7,11}$ que descrevem a sua utilização foram publicadas no idioma português, facilitando a apropriação do método.

Tal apontamento pode estar também associado ao avanço globalizado da rede mundial de computadores nos últimos anos, o qual permitiu maior facilidade no acesso à informação, incluindo em outros idiomas. Esta realidade viabilizou a disseminação do conhecimento e sua expansão por meio do acesso rápido e prático da internet..$^{12}$ Acredita-se que este avanço contribuiu, então, para o maior número de publicações utilizando o método da TFD, bem como a acessibilidade do conhecimento do método por parte dos pesquisadores brasileiros.

A enfermagem brasileira tem buscado a consolidação dos Programa de Pós-Graduação por meio de pesquisas, produções científicas de importância significativa e recrutamento de mestres e, principalmente, doutores qualificados para compor o corpo de conhecimento dessa disciplina científica. Nesse contexto, insere-se o resultado deste estudo documental, cujas pesquisas concentram-se nos cursos de doutoramento e mestrado acadêmico. Tal avanço contribuiu para notoriedade e qualidade acadêmi- 
ca da profissão em nível nacional e internacional, destacado ainda pela excelência na formação de doutores aptos a serem pesquisadores e cientistas. ${ }^{13}$

Além das aptidões descritas anteriormente, a construção de uma TFD exige do pesquisador denso conhecimento teórico dos elementos que a compõem, além de disposição e tempo para alcançá-la, o que pode explicar a predominância de pesquisas em nível de doutoramento, considerando que a formação do doutor oferece carga teórica e filosófica para o desenvolvimento do pensamento crítico e analítico, além de maior tempo para sua conclusão em detrimento do curso de mestrado.

Os resultados demonstraram que a utilização da TFD, apesar de mais frequente nas teses, é crescente em pesquisas de mestrado, pois o número entre teses e dissertações foi diretamente proporcional entre si. Tal realidade pode estar relacionada à disseminação do método e ao aumento do número de cursos de mestrado no território brasileiro. Porém, vale destacar que não há restrição quanto ao uso do método nos cursos de mestrado, entretanto, tal escolha demandará do pesquisador maior empenho para conseguir executá-lo, uma vez que o tempo do curso é menor para alcançar todas as etapas que lhe são exigidas.

Referente à disposição espacial das produções que utilizaram TFD pelas regiões federativas do Brasil, o predomínio do método nas regiões Sul e Sudeste, tanto em relação a IES como ao lócus do estudo, pode ser justificado pela maior concentração de Programa de Pós-Graduação em Enfermagem, como também por serem os mais antigos e de melhor avaliação da Coordenação de Aperfeiçoamento de Pessoal de Nível Superior (CAPES). Além disso, corrobora com outros estudos, ${ }^{14-16}$ nos quais estes programas são apresentados como substancialmente estáveis quando comparados a outros situados em diferentes regiões do país.

Dos relatórios de teses e dissertações analisados nesta pesquisa, $100 \%$ foram desenvolvidos por enfermeiros. De um lado, tal evidência pode relacionar-se aos critérios de inclusão proposto por este estudo, no qual foram incluídos apenas teses e dissertações de programas de enfermagem. Do outro modo, o crescente interesse de enfermeiros pelo método TFD para contribuir com a prática assistencial parece estar associada, uma vez que a formação de mestres e doutores nos referidos programas tem seguido uma perspectiva interdisciplinar, permitindo o ingresso de profissionais não enfermeiros.

No que concerne à atividade processual Assistência, esta apresentou iminente destaque para investigações utilizando a TFD como método. Tal fato pode ser compreendido considerando o cuidado centrado na pessoa humana, a essência da enfermagem, a quem se oferta cuidado, o ser que cuida, suas inter-relações e a gestão de cuidados. ${ }^{17}$ Nessa perspectiva, levanta-se a importância de estudos que tragam evidência à pesquisa, gerência e ensino como objetos de pesquisa utilizando o método da TFD para a enfermagem.

Ainda em relação à ênfase nas produções atreladas ao componente assistencial, esta pode ser justificada considerando o cuidado de enfermagem a essência da profissão. Adicionalmente é possível correlacionar seu destaque com a escolha do referencial metodológico straussiano, presente na maioria dos estudos, por estar fortemente relacionados com a experiência do pesquisador em relação ao fenômeno estudado. 6,8

Na abordagem teórica aos objetos pesquisados, o Interacionismo Simbólico foi o referencial adotado pela maioria das teses e dissertações e a sua escolha pode estar vinculada ao aspecto histórico de elaboração do método, uma vez que a Anselm Strauss, um dos seus idealizadores, buscava compreender fenômenos qualitativamente à luz do Interacionismo Simbólico. ${ }^{7}$

O referencial em apreço se propõe a compreender as reações do ser humano diante do significado de tudo que se apresenta para ele, o sentido que os objetos passam a desenvolver diante de sua relação com outros seres humanos e, por fim, as modificações de sentido que sofrem por meio de interpretações feitas pela pessoa diante da vivência de algumas situações. ${ }^{18}$

Outro referencial teórico adotado que merece destaque é a Teoria da Complexidade. Acredita-se que a utilização deste referencial na TFD devido à busca de conexões e inter-relações entre os conflitos que permeiam um fenômeno a ser estudado. Nesse contexto, Edgar Morin, principal pensador da complexidade, refere em sua teoria o homem como um ser multifacetado, não fragmentado e não simplificado, o qual resulta de um pensamento que inter-relaciona os acontecimentos na busca de explicações para eles. ${ }^{19}$

No que tange ao referencial metodológico adotado, no presente estudo, observou-se que não houve consenso em sua escolha. Este achado concorda com os relatórios nacionais de pesquisas brasileiras em que se apontam discordâncias nos processos analíticos, assim como em sua estrutura formal. Tal condição pode ser justificada em virtude de oposições metodológicas existentes entre os 
idealizadores do método. ${ }^{7-8}$

O método straussiano foi a abordagem mais utilizada nas teses e dissertações da amostra, apresentando o paradigma pós-positivista como norteador da sua construção teórica. A questão de pesquisa sobre sua ótica deve emergir diante da experiência do pesquisador e uma leitura aprofundada da temática. A análise dos dados acontece em três etapas de codificação e a validação do modelo teórico, que confere maior rigor científico, ${ }^{6,8}$ por apresentar etapa adicional de reflexão sobre os dados, articulando a participação de avaliadores com expertise no tema e/ou no método.

A metodologia clássica também foi descrita como referencial metodológico de escolha para a realização da TFD nas investigações do presente artigo. O método ancora-se no paradigma positivista, proposto por Glaser, ${ }^{11}$ que se propunha estudar fenômenos do indivíduo utilizando métodos exatos, quantitativos, onde as questões de pesquisa surgem por meio de dados emergentes sem aprofundamento da literatura sobre a temática. Neste método, a análise dos dados também ocorre por meio de codificação e na etapa de validação teórica é defendida a possibilidade de modificação de uma teoria à medida que novos dados surgem. ${ }^{8,11}$

Quanto aos recursos analíticos, a coleta de dados teve o uso da entrevista como mecanismo de coleta. Esta realidade confirma o fato de a entrevista ser o recurso predominantemente utilizado na TFD. ${ }^{8}$ A preferência substancial pelo método de entrevista, principalmente do tipo semiestruturada, na coleta de dados da TFD, deve-se também pela possibilidade de o pesquisador modificar o curso da coleta, aprofundando o entendimento com perguntas sobre o fenômeno em questão de acordo com a exigência dos dados. Assim, este método permite ao pesquisador percorrer diferentes caminhos durante a coleta. ${ }^{20}$ Destarte, é por meio da entrevista que se alcança a fidedignidade do que está sendo dito, pois é possível perceber o comportamento do participante durante a coleta de dados. ${ }^{10}$

Ademais, ressalta-se que houve tendência superior por parte dos estudos em adotar mais de um recurso para coleta de dados. Acredita-se que tal fato ocorra por permitir uma maior segurança na qualidade dos achados em investigações que utilizam diferentes técnicas de coleta. Isto porque seriam formas distintas de obter informações sobre o mesmo fenômeno, o que garantiria maior respaldo ao estudo.

Além disso, salienta-se que todos os recursos metodológicos adotados para coleta de dados com- binada estavam ligados a perspectiva straussiana, não havendo nenhum estudo com abordagem construtivista, apesar deste também incentivar o uso de múltiplas fontes de coleta de dados. ${ }^{8}$ Contudo, o banco de dados do CEPEn depende que os Programas de Pós-Gradução enviem as teses e dissertações para compor seus volumes.

Destacam-se ainda outras técnicas de coleta de dados como observação, grupos focais, análises de documentos, entrevista em grupo. Salienta-se que a utilização destes recursos é entendida como possibilidade e não como mecanismo inflexível e rígido para coletar dados. Deste modo, o investigador deve ter o conhecimento apropriado sobre o método e a vertente que será utilizada com vistas a evitar possíveis equívocos nos resultados do estudo. ${ }^{21}$

O expressivo número de estudos que utilizou a observação como estratégia de coleta de dados pode ser esclarecido por este método permitir o entendimento daquilo que não é dito, seja por não existir vocabulário ou pela incapacidade do participante em expressar verbalmente o desejado. ${ }^{10}$

Identificou-se nos achados das teses e dissertações em questão que, dentre os tipos de observações existentes, a não participante foi a escolha da maioria dos autores. A opção por esta técnica de coleta pode ter relação com o fato de o pesquisador estar presente no contexto do objeto em estudo, mas ter somente o papel visual, observando assim os indivíduos da pesquisa sem colaborar com as informações. Contudo, enfatiza-se que independentemente do tipo de observação adotada, o pesquisador deve registrá-las metodicamente. ${ }^{8}$

É importante considerar que as escolhas das técnicas para coleta de dados se apresentaram coerentes com as recomendações e especificidades das perspectivas metodológicas, uma vez que foram utilizados recursos como entrevistas, observações, análise de documentos e grupo focal.

Faz-se necessário enfatizar que o objetivo principal da TFD é buscar os significados das relações sociais, interações e associações que são sinalizadas a partir dos significados atribuídos pelos sujeitos que as vivenciam e são capazes de explicitar seus movimentos, e não apenas significados existenciais para o ser pessoa, para o seu viver e relacionados à imagem que a pessoa projeta ou que representa para ela. Assim, diferencia-se de um estudo fenomenológico ou de representações sociais, ou de outros que também buscam a compreensão da essência do fenômeno ou condição. ${ }^{8}$

Neste sentindo, a pesquisa documental apontou, em sua maioria, o uso adequado dos recursos 
analíticos da TFD, em detrimento de poucos que não foram realizados efetivamente. Em relação a descrição da construção dos grupos amostrais, a maioria das pesquisas descreveram com êxito a seleção dos sujeitos para compor a amostra, o que foi detalhado na metodologia de cada pesquisa. Somente uma pequena parcela dos estudos não enfatizou a descrição do grupo amostral, campo essencial na metodologia de investigação e de ênfase na seleção desses sujeitos que estejam vivenciando a situação social que se está estudando, ou seja, aqueles que têm informações ou conhecimento sobre o estudo em questão. Considerando que os grupos amostrais são construídos a partir de hipóteses desenvolvidas pelo processo indutivo eda análise dos dados, estes configuram-se como importante estratégia para o desenvolvimento de conceitos amplos e embasados nos dados. A operacionalização inadequada poderá comprometer os elementos da teoria. ${ }^{21}$

Sobre o desenvolvimento desses recursos, também é importante destacar o efetivo cumprimento das etapas do método em que a coleta e análise dos dados foram realizadas simultaneamente para elucidação e verificação dos dados, pois, os pesquisadores precisam apropriar-se do processo de raciocínio indutivo e dedutivo para estabelecer os conceitos por meio de habilidades que envolvam sensibilidade e criatividade, objetivando compreensão complexa e detalhada do objeto abordado. ${ }^{11,22}$

A codificação teórica, ao estabelecer novas conexões e transformá-las em ideias relevantes, determina o caráter original da teoria. $\mathrm{O}$ esforço do pesquisador nessa fase analítica é no sentido de delimitar os atributos, que são designados por Strauss e Corbin como propriedades, com a finalidade de detalhar o conteúdo das categorias. Isso é, os conceitos avançam e consolidam-se em termos de propriedades e dimensões. ${ }^{8}$

Ao analisar as etapas de codificação, destacase que a maioria das pesquisas descreveram detalhadamente as etapas segundo definido no método. Entretanto, pequena parcela, deixou lacunas na descrição das etapas de codificação, ou não descreveram em seu método de acordo com a perspectiva metodológica adotada.

Strauss e Corbin apresentam uma abordagem estruturada e sistemática para a análise dos dados em três etapas. A codificação aberta, como a primeira etapa do processo de análise. A segunda etapa é a codificação axial que objetiva desenvolver sistematicamente as categorias e relacioná-las, e a última etapa é a codificação seletiva cujo objetivo é integrar e refinar categorias em um modelo analí- tico. ${ }^{6}$ Já Glaser defende a realização da codificação teórica e descarta a necessidade da codificação axial, uma vez que conecta novamente os elementos fragmentados. ${ }^{11}$ Dessa forma, nos processos de análise, a codificação teórica difere da substantiva com relação ao nível e tipo de abstração. Charmaz preconiza que o processo de codificação para análise dos dados seja realizado em pelo menos duas etapas: codificação inicial e codificação focalizada. ${ }^{7,23}$

De acordo com as diferenças entre as etapas de codificação de cada perspectiva metodológica da TFD, é importante registrar com especificidade o modo como foi utilizado o método de acordo com o referencial teórico-filosófico que norteou a pesquisa, visto que a utilização isolada das etapas de codificação da TFD para analisar os dados coletados não caracteriza o método como técnica ou método de análise.

As perspectivas metodológicas da TFD sugerem também a utilização de diagramas e memorandos como estratégia para orientar o pesquisador ao longo da realização da pesquisa. Tanto o diagrama, que contribui para apresentar visualmente as categorias e suas conexões ao longo do processo de pesquisa, quanto o memorando, registros que contêm produtos de análise e objetivam o desenvolvimento de conceitos, ${ }^{7-8}$ foram bem explorados nas pesquisas.

Embora os autores do método da TFD não expressem a obrigatoriedade no uso desses recursos, a sua utilização é importantíssima para determinar as relações entre os conceitos e colaborar na construção das hipóteses de pesquisa. Destacando-se o uso dessas ferramentas para auxiliar na análise e construção de hipóteses e conceitos, visto que são importantes estratégias analíticas, considerados registros da análise, os quais podem ser feitos manualmente ou por meio de softwares para análise de dados qualitativos. ${ }^{6}$

No que tange à etapa de apresentação da teoria, houve expressivo número de trabalhos que realizou tal etapa, a qual visa demonstrar que o modelo teórico é representativo em relação à realidade investigada, porém um número reduzido de trabalhos não apresentou nomeadamente os resultados como teoria substantiva, implica em leitura e aplicabilidade, a outros contextos de tempo e espaço, não permitindo modificações e incorporações de novos elementos que visem ao aprimoramento dos conhecimentos relativos ao fenômeno que foi investigado. ${ }^{8}$ Por fim, todas as etapas de construção de uma TFD ancoradas nos recursos analíticos bem apropriados, levam a validação e apresentação da teoria construída, com as categorias e suas relações 
até atingir o fenômeno ou categoria central, por profissionais experts no método ou na temática em estudo e/ou ao grupo ou parte do grupo de participantes da pesquisa.

Ainda, aponta-se que, embora fosse pequeno número de dissertações e teses não localizados no formato texto completo, este representou limitação metodológica. Essa limitação pode ser relacionada à opção de pesquisar somente no CEPEn/ABEn e nos repositórios das IESs.

\section{CONCLUSÃO}

O estudo buscou contribuir com a identificação dos recursos analíticos da TFD em teses e dissertações vinculadas aos programas de Pós-Graduação de Enfermagem no Brasil e mostrar como este método vem sendo utilizado pelos pesquisadores, com o intuito de subsidiar a construção do conhecimento desse método de forma confiável e fiel a suas etapas.

A pesquisa aos documentos buscou verificar principalmente a coerência ao método adotado nos estudos selecionados, estimulando a reflexão sobre a importância de produções de qualidade, bem como agregar rigor ao desenvolvimento de conhecimento à disciplina da Enfermagem, a fim de contribuir com a continuidade do seu aprimoramento. Portanto, no conjunto da produção em enfermagem identificada nesta pesquisa, foram poucas as dissertações e teses que apresentaram o não cumprimento de descrição detalhada em relação à aplicação da TFD, revelando que ainda há fragilidades ou necessidade de aperfeiçoamento quanto ao manejo do método.

Destacam-se os pontos de dificuldade na seleção da amostra da pesquisa, como: a escassez de informações importantes nos resumos; teses e dissertações não disponibilizadas na íntegra nos repositórios institucionais que, em geral, tratavamse de produções mais antigas; e o reduzido número de trabalhos que discutem as suas limitações metodológicas com relação ao uso da TFD.

Por todos esses aspectos mencionados, a pesquisa permitiu detectar que para a construção de uma TFD, é preciso conhecimento teórico dos principais elementos que constitui esse método, além de dedicação e tempo do pesquisador para que se cumpram todos os processos visando alcance da teoria.

\section{REFERÊNCIAS}

1. Santos SVM, Ribeiro ME, Motta ALC, Silva LJA, Resck ZMR, Terra FS. Building knowledge in nursing: a reflective theoretical and methodological approach for nurses training. J Nurs UFPE on line [Internet]. 2015 [cited 2017 Jun 23]; 10(1):172-8. Available from: http:/ / www.revista.ufpe.br/revistaenfermagem/ index.php/revista/article/view/8446/pdf_93742

2. Scochi CGS, Munari DB, Gelbcke FL, Erdmann AL, Gutiérrez MGR, Rodrigues RAP. Pós-graduação stricto sensu em enfermagem no Brasil: avanços e desafios. Rev Bras Enferm [Internet]. 2013 [2017 Jun 23]; 66(esp):80-9. Available from: http:/ / www.scielo. $\mathrm{br} / \mathrm{pdf} /$ reben/v66nspe/v66nspea11.pdf

3. Andrews, T. Ontological issues in qualitative researsh in nursing. Texto Contexto Enferm [Internet]. 2016 [cited 2016 Oct 08]; 25(3):editorial. Available from: http://www.scielo.br/pdf/tce/v25n3/0104-0707tce-25-03-editorial.pdf

4. Medeiros M. Thinking about qualitative research. Rev Eletr Enf [Internet]. 2012 [cited 2016 Jul 25]; 14(2):224-5. Available from: https:// revistas.ufg.br/ fen/article/view/13628/11616

5. Minayo MCS, Guerriero ICZ. Reflexividade como éthos da pesquisa qualitativa. Ciênc Saúde Coletiva [Internet]. 2014 [cited 2017 Jun 19]; 19(4):1103-12. Available from: http://www.scielo.br/pdf/csc/ v19n4/1413-8123-csc-19-04-01103.pdf

6. Strauss A, Corbin J. Pesquisa qualitativa: técnicas e procedimentos para o desenvolvimento de teoria fundamentada. $2^{\mathrm{a}}$ ed. Porto Alegre (RS): Artmed; 2008.

7. Charmaz K. A construção da teoria fundamentada: guia prático para análise qualitativa. Porto Alegre (RS): Artmed; 2009.

8. Santos JLG, Erdmann AL, Sousa FGM, Lanzoni GMM, Melo ALSF, Leite JL. Methodological perspectives in the use of grounded theory in nursing and health research. Esc Anna Nery [Internet]. 2016 [cited 2016 Jul 25]; 20(3):e20160056. Available from: http:/ / www.scielo.br/pdf/ean/v20n3/en_1414-8145ean-20-03-20160056.pdf

9. Teodosio SSS, Silva ER, Padilha MI, Mazera MS, Borenstein MS. Oral history and documental investigation as a research itinerary in nursing: a bibliometric study (2000-2014). Esc Anna Nery [Internet]. 2016 [cited 2017 Jun 23]; 20(4):e20160087. Available from: http://www.scielo.br/pdf/ean/ v20n4/en_1414-8145-ean-20-04-20160087.pdf

10. Gomes IM, Hermann AP, Wolff LDG, Peres AM, Lacerda MR. Grounded theory in nursing: integrative review. J Nurs UFPE on line [Internet]. 2015 [cited 2016 Jul 25]; 9(1):466-74. Available from: http:/ / www. revista.ufpe.br/revistaenfermagem/index.php/ revista/article/view/5380/pdf_7072

11. Glaser BG. The grounded theory perspective: conceptualization contrasted with description. Mill Valley, CA (EUA): Sociology Press; 2011.

12. Milanesi L. Biblioteca pública: do século XIX para o XXI. Rev USP [Internet]. 2013 [cited 2016 Jul 23]; 97(2):59-70. Available from: http://www.revistas. 
usp.br/revusp/issue/view/5058

13. Scochi CGS, Munari DB, Gelbcke FL, Ferreira MA. The challenges and strategies from graduate programs innursing for the dissemination of scientific production at international journals. Esc Anna Nery [Internet]. 2014 [cited 2017 Jun 21]; 18(1):5-6. Available from: http://www.scielo.br/pdf/ean/v18n1/ en_1414-8145-ean-18-01-0005.pdf

14. Pizzani L, Lopes JF, Manzini MG, Martinez CM. Bibliometric analysis of theses and dissertations on prematurity in the Capes database. J Pediatr (Rio J) [Internet]. 2012 [cited 2016 jul 20]; 88(6):479-82. Available from: http://www.scielo.br/pdf/jped/ v88n6/v88n06a06.pdf

15. Salvador PTCO, Rodrigues CCFM, Lima KYN, Alves KYA, Santos VEP. Non-conventional technologies for data collection in Brazilian dissertations and theses. Rev Bras Enferm [Internet]. 2015 [cited 2016 jul 21]; 68(2):269-77. Available from: http:/ / www.scielo.br/ pdf/reben/v68n2/0034-7167-reben-68-02-0269.pdf

16. Ferreira MA. The classical and the emerging: challenges in the production, dissemination and use of Nursing knowledge. Rev Bras Enferm [Internet]. 2013 [cited 2017 Jun 21]; 66(spe):45-50. Available from: http://www.scielo.br/pdf/reben/v66nspe/ v66nspea06.pdf

17. Santos JLG, Pestana AL, Guerrero P, Meirelles BSH, Erdmann AL. Nurses' practices in the nursing and health care management: integrative review. Rev. Bras. Enferm [Internet]. 2013 [cited 2017 Jun 21]; 66(2):257-63. Available from: http:/ / www.scielo.br/ pdf/reben/v66n2/16.pdf

18. Martins CBC. O legado do Departamento de Sociologia de Chicago (1920-1930) na constituição do Interacionismo Simbólico. Rev Sociedade e Estado [Internet]. 2013 [cited 2017 Jun 18]; 25(2):217-39. Available from: http://www.scielo.br/pdf/se/ v28n2/v28n2a03.pdf

19. Cruz RAO, Araújo ELM, Nascimento NM, Lima RJ, França JRFS, Oliveira JS. Reflections in the light of the complexity theory and nursing education. Rev Bras Enferm [Internet]. 2017 [cited 2017 Jun 23]; 70(1):224-7. Available from: http://www.scielo.br/pdf/reben/ v70n1/en_0034-7167-reben-70-01-0236.pdf

20. Cunha JJ, Santos PMD, Correa ABH, Hermann AP, Lacerda MR. Opportunity to work with the grounded theory in nursing undergraduate course data. Cienc Cuid Saude [Internet]. 2012 [cited 2016 Jul 25]; 11(3):593-9. Available from: http://dx.doi. org/10.4025/cienccuidsaude.v11i3.14183

21. Furlanetti MRR, Barros NF. Construção da Teoria Fundamentada: guia prático para análise qualitativa. Ciênc. saúde coletiva [Internet]. 2013 [cited 2016 Jul 21]; 18(1):283-4. Available from: http:/ / www.scielo.br/pdf/csc/v18n1/29.pdf

22. Creswell JW. Investigação Qualitativa e Projeto de Pesquisa: escolhendo entre cinco abordagens. $3^{\mathrm{a}}$ ed. Porto Alegre (RS): Penso; 2014.

23. Engward H. Understanding grounded theory. Nurs Stand [Internet]. 2013 [cited 2016 Jul 15]; 28(7):3741. Available from: http://dx.doi.org/10.7748/ ns2013.10.28.7.37.e7806
Correspondência: Glauber Weder dos Santos Silva Universidade Federal do Rio Grande do Norte, Campus Universitário Lagoa Nova, Departamento de Enfermagem, s/n, 59078-907 - Lagoa Nova. Natal, RN, Brasil

E-mail: glauberweder@hotmail.com
Recebido: 11 de outubro de 2016 Aprovado: 27 de junho de 2017

This is an Open Access article distributed under the terms of the Creative Commons (CC BY). 